\title{
Peter Custers
}

\section{„Disparater Tausch" Diamanten, Öl und Bürgerkrieg in Afrika}

\section{Einleitung: der Weltbankreport}

Im Juni 2000 veröffentlichte die Weltbank einen Bericht über die Bürgerkriege in den Ländern des Südens, der von Seiten der internationalen Presse hohe Aufmerksamkeit erfuhr. Der Bericht stammte von Paul Collier, der die Forschungsgruppe zur Entwicklungspolitik bei der Weltbank leitet. Er entwirft eine „Theorie der räuberischen Tätigkeit“, die sich als Interpretation der Bewegungen des bewaffneten Widerstands aus ökonomischer Sicht anpreist. Der Bericht argumentiert folgendermaßen:

„Erst die Möglichkeit Raubzüge durchzuführen, bestimmt auch das Risiko von Konflikten (sprich: bewaffneten Konflikten). Die Räuberei selbst mag nur als bedauerliche Notwendigkeit auf dem Wege zur empfundenen Gerechtigkeit oder Macht daherkommen, die Bedingungen für räuberische Tätigkeit sind jedoch entscheidend. Ob ein Konflikt durch räuberische Tätigkeit motiviert, oder dadurch nur ermöglicht wird, führt zur selben Schlußfolgerung: Eine Rebellion steht in keiner Beziehung zu objektiven Mißständen, solange sie durch die Möglichkeit räuberischer Tätigkeit verursacht wird“ (Collier 2000).

Ganz unabhängig von den Zielen der Rebellen, die den bewaffneten Widerstand in Gang setzen oder aufrecht erhalten, argumentiert Collier, ist es aus volkswirtschaftlicher Sicht letztendlich der Zugang zu Rohmaterialien, beispielsweise Diamanten, der primär die Richtung der Ereignisse bestimmt.

Colliers Bericht weist schon beim ersten Lesen zahlreiche Fehler auf. Zunächst einmal basiert er auf diversen falschen Dichotomien und Pauschalisierungen. Er konstatiert, daß Rebellenorganisationen wie jene in Sierra Leone und Angola sich vollständig auf den Export von Edelsteinen und anderen natürlichen Ressourcen zur Finanzierung ihrer Kriegsführung verlassen. Allerdings begnügt sich der Bericht nicht damit, die Rebellen in Sierra Leone und Angola zu verurteilen, vielmehr bemüht er diese Beispiele, um alle Guerillaaktivitäten zu kriminalisieren, indem er sie alle als „Manifestationen des organisierten Verbrechens" bezeichnet (Collier 2000). Diese Pauschalisierung entbehrt offensichtlich jeglicher Grundlage, da sie die unterschiedlichen sozialen und ökonomischen Ausgangsbedingungen der Guerillabewegungen ignoriert. Indem er auf den Unterschied zwischen (unbewaffnetem) Protest und 
(bewaffneten) Rebellenbewegungen abhebt, wird eine völlig falsche Dichotomie nahegelegt, insofern unterstellt wird, der gewaltlose Protest speise sich tatsächlich aus sozialen Mißständen, was bei militanten Bewegungen hingegen nicht der Fall sei. Außerdem ignoriert Collier mit der Behauptung, sein Bericht stehe in „geringer oder keinerlei Beziehung zu Kriegen, die von Regierungen geführt werden“, zusätzlich die Tatsache, daß kriegerische Auseinandersetzungen unter Regierungsbeteiligung in Afrika und anderswo ebenfalls sehr häufig von Rohstoffexporten abhängig sind. Ein weiterer Mangel von Colliers Analyse besteht darin, dass er sie als ökonomische Analyse präsentiert, während sie nichts weiter als eine mechanische Übung in Statistik darstellt. Obwohl der Autor die Aufmerksamkeit zu Recht auf eine vernachlässigte Thematik lenkt, unternimmt er es an keiner Stelle seines Berichts, der über die „wirtschaftlichen Ursachen von Bürgerkriegen“ handeln soll, die Rolle externer Wirtschaftskräfte wie etwa der transnationalen Unternehmen (TNCs) in den Bürgerkriegen der südlichen Kontinente zu analysieren. Obgleich Collier eine Thematik aufgreift, für die sich lange Zeit vorwiegend kapitalismuskritische Ökonomen interessierten, beispielsweise die Abhängigkeit der Volkswirtschaften des Südens vom Export (einzelner) Rohstoffe, verharmlost er die Tatsache, daß die Profite aus diesen Exporten hauptsächlich die TNCs bereichern, die im Norden angesiedelt sind. Collier erhebt Anklage gegen alle bewaffneten Rebellenbewegungen, übergeht aber die Verantwortung, welche die TNCs für Kriegsverbrechen tragen, die in Angola, Sierra Leone und anderen südlichen Ländern auf der Basis eines Handelssystems begangen werden, das es den Kriegsparteien ermöglicht, Rohmaterialien gegen Waffen einzutauschen.

Nichtsdestotrotz glaube ich, daß der Weltbankbericht kritischen Volkswirtschaftlern und politischen Aktivisten als eine Anregung dienen sollte. Schließlich kommt Collier mit seiner schlechten Analyse zum Teil auch deswegen davon, weil es an einer systematischen Analyse der Verbindung zwischen nördlichen Waffenexporten und südlichen Rohstoffexporten seitens der Linken bisher mangelt. Dieser Austausch von nutzlosen oder gar zerstörerischen Produkten (wie Waffen) gegen natürliche Reichtümer soll im folgenden als Disparater Tausch bezeichnet werden. Obwohl dieses System in den letzten dreißig Jahren Anwendung gefunden hat, haben fortschrittliche Volkswirtschaftler es meines Wissens bis heute nie theoretisch erfaßt, ganz im Gegensatz beispielsweise zur Biopiraterie (Shiva 1998). Insofern sollte das Erscheinen des fragwürdigen Weltbankberichts als Erinnerung daran dienen, daß die Formulierung einer Theorie des Disparaten Tausches seit langem überfällig ist. Im folgenden will ich die Notwendigkeit einer solchen Theorie für das Verständnis der gegenwärtigen Kriege in Afrika aufzeigen. 


\section{Diamanten und die Finanzierung des angolanischen Bürgerkriegs}

Das Rohmaterial, welches neuerdings den höchsten Symbolgehalt für die Finanzierung von Bürgerkriegen im Süden eingenommen hat, sind Diamanten. Die Bezeichnung „Blutdiamanten“ bezieht sich auf jene Funktion der wertvollen Steine, die als Luxusgüter an wohlhabende Konsumenten im Norden verkauft zu werden. Sie kommen aus Gegenden in Afrika, die von Rebellen kontrolliert werden. Zum Zeitpunkt der Veröffentlichung von Colliers Bericht beherrschte die Debatte über die Blutdiamanten die Weltpresse an vorderster Stelle. Diese Stellung im Scheinwerferlicht der Öffentlichkeit verkörperte das zunehmende Unbehagen unter den politischen Verantwortlichen und den Trägern der öffentlichen Meinung des Nordens über die unmenschlichen Praktiken von De Beers und anderen Diamantengesellschaften. Obgleich diesen Unternehmen sehr wohl bekannt ist, daß der Erlös aus Diamantenverkäufen häufig der Waffenbeschaffung dient, schließen sie die Augen vor den Kriegsverbrechen ihrer Zulieferer. Einige der grauenvollsten Kriege, die heute in Afrika toben, können nur geführt werden, weil Rebellen große Diamantengebiete kontrollieren. Beispiele hierfür sind die Bürgerkriege in Sierra Leone und Angola, und zu einem geringeren Ausmaß der internationalisierte Konflikt im Kongo. Insofern ist das Beispiel der Blutdiamanten zentral für unsere Diskussion über den Disparaten Tausch im Zusammenhang mit Bürgerkriegen.

Obgleich sich nicht alle neueren Berichte über Blutdiamanten explizit dazu äußern, kann man davon ausgehen, daß es die angolanische Rebellenbewegung UNITA war, die die Nutzung von Diamanten zur Kriegsfinanzierung historisch institutionalisiert hat (Global Witness 1998; Smilie/Gberie/Hazleton 2000). Während bis zum Ende des Kalten Krieges die UNITA ihre kriegerischen Machenschaften dank der Finanzierung durch die USA aufrechterhalten konnte, war die Weiterführung ihres Krieges gegen die angolanische Regierung in den 90er Jahren nur mit Hilfe des Abbaus und Verkaufs von Diamanten möglich. Dies dokumentiert ein Bericht von Human Rights Watch von 1994 über den angolanischen Krieg und den internationalen Waffenhandel. Gleichzeitig wirft der Bericht ein scharfes Licht auf die systematischen und grauenvollen Verletzungen des Kriegsrechts durch die UNITA in der Periode, die in Angola den Wahlen von 1992 folgte (deren Ergebnis die UNITA nicht anerkannte) - und die Tatsache, daß offizielle Vertreter von De Beers genau während dieser Periode Edelsteine aus Rebellengebieten kauften. ${ }^{1}$

1 Der Bericht stellt unter anderem klar: „Das De Beers Diamantenkartell und andere internationale Händler erwarben Edelsteine, die unter Verletzung angolanischer Gesetze auf Gebieten abgebaut wurden, die unter Kontrolle der Rebellen standen. Zwischenhändler haben für den Diamantenschmuggel über die Südgrenze von Zaire Zahlungen von mehreren Hundert- 
Das Beispiel der methodischen Anwendung des Disparaten Tauschs durch die UNITA ist auch unter quantitativen Gesichtspunkten höchst signifikant, denn die Einnahmen, die sie aus Diamantenverkäufen angeblich hat, sind immens. Der Global Witness Bericht $A$ Rough Trade zitiert Zahlen, denen zufolge sich diese Einnahmen in den Jahren 1992 bis 1998 zwischen 200 und 700 Mio. Dollars jährlich bewegten. So beliefen sich 1993, als ein Teil der schlimmsten Kämpfe des gesamten Konflikts stattfand, bei denen in einem Zeitraum von fünf Monaten 182.000 Menschen umkamen, die Einnahmen der UNITA aus dem illegalen Diamantenexport auf 600 Mio. Dollar (Global Witness 1998). Global Witness schätzt, daß die UNITA in den sechs Jahren von 1992 bis 1998 insgesamt etwa 3,72 Mrd. Dollar aus dem Diamantenhandel eingenommen hat (ebd.). ${ }^{2}$ So überrascht es nicht, daß die Rebellenorganisation während der 90er Jahre in der Lage war, die Beschaffung hoch entwickelter Waffensysteme auf dem internationalen Markt fortzusetzen, inklusive Panzerminen und Raketenwerfer (Human Rights Watch 1994: 47; Hagelin/Wezeman 2000). Zusätzlich ist das Beispiel der Anwendung des Disparaten Austauschs durch die UNITA so fernab jeglicher Kontroverse, daß es sogar Eingang in die Resolutionen gefunden hat, die der Sicherheitsrat der Vereinten Nationen anläßlich des andauernden angolanischen Bürgerkrieges verabschiedet hat. In der Resolution 864 vom September 1993, verbietet der Sicherheitsrat den Verkauf und die Lieferung jeglicher Waffen und jeglichen diesbezüglichen Materials oder militärischer Unterstützung an die UNITA und verlangt strenge Disziplin seitens seiner Mitglieder, um die effektive Umsetzung des Embargos zu gewährleisten (Human Rights Watch 1994: 48). In einer erneuten Resolution, die fast 5 Jahre später verabschiedet wurde, Resolution 1173 vom Juni 1998, legte der Sicherheitsrat einen Bann auf alle Diamantenexporte aus Angola, die über kein offizielles Regierungszertifikat verfügten, und verbot alle Verkäufe von Abbauausrüstung und/oder Abbaudienste an die UNITA (vgl. auch Global Witness $1998 \mathrm{zu}$ den beiden Resolutionen). Obgleich es bedauerlich ist, daß die Diamantenfrage nicht

tausend Dollar an UNITA-Funktionäre geleistet. Im Januar 1993 soll UNITA-Funktionären von Händlern in der südlichen Stadt Tshipaka, ungefähr 70 Meilen von der angolanischen Grenze entfernt, 400.000 Dollar in bar ausbezahlt worden sein. De Beers schloß sein Büro in Tshipaka im Januar 1993 aufgrund der 'Unsicherheit', die Verbindung zu den lokalen Händlern wurde aber aufrecht erhalten. De Beers hat zugegeben, 1992 am 'Offenen Markt' 500 Mio. Dollar für legal und illegal abgebaute Diamanten mit Ursprung in Angola ausgegeben zu haben“ (Human Rights Watch 1994: 57/58).

2 Paul Collier (2000) behauptet: „Savimbi, dem Kopf der angolanischen Rebellenorganisation UNITA, wurde nachgesagt, 4 Mrd. Dollar in Aktiva während des ersten Kriegs akkumuliert zu haben. Einen Teil davon benutzte er dazu, den zweiten zu finanzieren“ und: „Diamanten hatten die UNITA so reich gemacht, daß nichts, was Geber anzubieten hatten, von Interesse war, wohingegen erneute Raubzüge massive Gewinne boten. Es wird vermutet, daß die UNITA in den ersten zwei Jahren des wieder aufgeflammten Krieges um die 2 Mrd. Dollar aus dem Diamantenabbau erbeutet hat" (Collier 2000: 18); allerdings ignoriert Collier völlig die Rolle von De Beers. Das Beispiel illustriert die Unfähigkeit des Autors, auch nur wahrzunehmen, daß TNCs hohe Gewinne aus der Fortsetzung von Bürgerkriegen erzielen. 
lutionen). Obgleich es bedauerlich ist, daß die Diamantenfrage nicht schon früher auf die Tagesordnung kam und gleichzeitig mit der Waffenfrage behandelt wurde, und obwohl beide Resolutionen bis zum heutigen Tag beständig verletzt wurden, ist doch die Tatsache, daß die Rolle des Disparaten Austauschs im angolanischen Bürgerkrieg durch UN Resolutionen betont wird, politisch bedeutsam.

\section{Die Struktur des internationalen Diamantenmarkts}

Um ein besseres ökonomisches Verständnis der Frage der Blutdiamanten zu vermitteln, müssen wir einen genaueren Blick auf den internationalen Diamantenmarkt werfen. Sein wichtigstes Merkmal ist, wie schon oft betont wurde, die Tatsache, daß er von einer einzigen transnationalen Gesellschaft beherrscht wird, dem südafrikanischen Konzern De Beers, der sowohl außerordentlichen Einfluß auf den weltweiten Abbau von Rohdiamanten ausübt, als auch gleichzeitig über Jahrzehnte hinweg eine strenge Kontrolle über die Vermarktung der Edelsteine. Einem detaillierten Bericht über Blutdiamanten aus Sierra Leone vom Januar 2000 zufolge, fördert De Beers aus eigenen Minen in Südafrika und in Partnerschaft mit den Regierungen von Botswana, Namibia und Tansania fünfzig Prozent der Edelsteindiamanten der Welt (dem Wert nach). Zusätzlich kauft De Beers Diamanten von „Außenmärkten" und verkauft sie über eine Verkaufsorganisation, die ihrer Kontrolle untersteht, die Central Selling Organisation (CSO) (Smilie/Gberie/Hazleton 2000). Obwohl die Zahlen über den genauen Prozentsatz der weltweiten Rohdiamanten, die über die CSO gehandelt werden, variieren, ist davon auszugehen, daß es sich um mindestens sechzig Prozent handelt (Roche 2000: 16). ${ }^{3}$

Der CSO wird nachgesagt, als Kartell zu arbeiten, was insofern richtig ist als sie den Marktpreis von Rohdiamanten manipuliert. ${ }^{4}$ Einerseits ist De Beers bestrebt, die Menge des Marktangebots an Rohdiamanten zu regulieren indem sie ihre eigene Vorratshaltung betreibt. Andererseits diktiert aber auch De Beers allein den Preis für die Diamantenpakete, die im CSO Hauptquartier in London zusammengestellt und über eine ausgesuchte Gruppe von 160 Zwischenhändlern, die sogenannten Sightholders, vertrieben werden. Beobachter sind sich einig, daß es der Gesellschaft über Jahrzehnte hin mit geringfügigen Einschränkungen möglich war, entscheidenden Einfluß auf den Preis

3 Marc Roche stellt fest, daß De Beers am Ende der 80er Jahre zwar fast die gesamte Diamantenproduktion der Welt absorbierte, daß sich aber in letzter Zeit einige Staaten aus der Kontrolle der Gesellschaft befreien konnten und so den Zugriff von De Beers auf den Diamantenabsatz reduzierten.

4 Mandel (1974: 402) listet Kartelle unter den verschiedenen Formen der Kapitalkonzentration auf, betont aber, daß Kartelle Gruppierungen sind, innerhalb derer die partizipierenden Unternehmen ihre Unabhängigkeit wahren. 
für Rohdiamanten auf dem Weltmarkt auszuüben und es ist bekannt, daß durch die Manipulation von Angebot und Nachfrage künstlich hohe Marktpreise unterstützt wurden (Smilie/Gberie/Hazleton 2000: 25). Obgleich sich die Monopolstellung von De Beers über den internationalen Diamantensektor zu lockern scheint, ist es wenig wahrscheinlich, daß die Gesellschaft ihre doppelte Kontrolle über den Abbau und die Vermarktung der Edelsteine aufgeben wird - so lange nicht bis Konkurrenten, Kampagnen und die politisch Verantwortlichen sie dazu zwingen.

Die Rolle von De Beers legt einen Vergleich mit der Situation auf dem internationalen Ölsektor nahe. Sowohl auf dem Rohölmarkt als auch auf dem Markt für Rohdiamanten existiert ein monopolistischer Kontrollmechanismus zum Zweck der Preisfestlegung, im ersten Fall das Kartell erdölproduzierender Länder, und im zweiten Fall eine einzige transnationale Abbaugesellschaft. Diese Ähnlichkeit der zwei Sektoren mag seinen Ursprung in der Tatsache finden, daß beide aus einem System des Disparaten Tauschs hervorgegangen sind. Gleichzeitig entblößt dieser Vergleich aber auch die Tatsache, daß der Druck, den die südlichen Staaten über Angebot und Preise auf dem internationalen Diamantensektor ausüben, relativ gering ist. Während die südlichen Produzentenländer, die ihre Kräfte in der OPEC vereint haben, gemeinschaftlich den Marktpreis für Öl festlegen, übernimmt dies im Fall der Diamanten eine einzige transnationale Gesellschaft, die die peripheren Volkswirtschaften dominiert. Insofern ist auch die Gefahr, daß periphere Länder im Zuge des Disparaten Austauschs von Diamanten gegen Waffen ausbluten, besonders hoch.

Die Theorie des Disparaten Tauschs zwingt uns zu der Frage, in welchem Ausmaß die Fortsetzung der grauenvollen Bürgerkriege in Angola und andernorts in Afrika untrennbar mit der Existenz monopolistischer Kontrolle über den Diamantensektor zusammenhängt. Kürzlich wurden in Form von Empfehlungen im Rahmen von Forschungsberichten, Ratschlägen eines UNExpertenforums etc. verschiedene Regulationsmechanismen vorgestellt, mittels derer Blutdiamanten aus dem internationalen Handel entfernt werden sollen. Einige dieser Vorschläge beinhalten die Einführung eines Herkunftszertifikats, um den Schmuggel von Rohdiamanten aus Gebieten, die sich unter Kontrolle von Rebellen befinden, zu unterbinden (Smilie/Gberie/Hazleton 2000; IISS 2000). Daß der Vorschlag, ein Zertifizierungssystem einzurichten, vom Welt Diamanten Kongreß im Juli letzten Jahres angenommen wurde, wurde als größeres Zugeständnis seitens De Beers angesehen. Trotzdem werden ohne eine vollständige Umgestaltung des Diamantensektors weiterhin die produzierenden Länder, besonders in Afrika, zu den Opfern des manipulativen Vorgehens von De Beers gehören und einen außerordentlich schweren Stand haben, diese Versklavung unter dem System des Disparaten Tauschs zu durchbrechen. 


\section{Öl und die Finanzierung des angolanischen Bürgerkriegs}

Ein weiteres strategisch unerläßliches Rohmaterial in Afrikas Bürgerkriegen stellt Öl dar, und auch hier ist es außerordentlich lehrreich, den angolanischen Fall zu diskutieren. Die Erfahrung, die die angolanische Regierung mit dem Disparaten Tausch gesammelt hat, ähnelt in gewisser Hinsicht sehr stark denen nahöstlicher Staaten, denn schließlich gibt es mehr als nur eine Verbindung zwischen den angolanischen Ölexporten und dem Waffenimport, um die UNITA zu bekämpfen. Zunächst einmal besteht eine budgetmäßige, d.h. indirekte Korrelation zwischen den beiden Handelsströmen. Einerseits ist das Öl für über $90 \%$ der Exporteinnahmen des Landes verantwortlich. In den 90er Jahren erzielte der angolanische Staat ein jährliches Öleinkommen zwischen 1,8 und 3 Mrd. US-Dollar. Wie Global Witness berichtet, sorgte das Öl für den Großteil der Staatseinnahmen während des vergangenen Jahrzehnts. Andererseits besagen offizielle Daten, daß „Verteidigung und öffentliche Ordnung“ den größten Posten bei den Staatsausgaben einnehmen. In den Jahren zwischen 1994 und 1997 schwanken die Ausgaben Berichten zufolge um die 35\%-Marke (Global Witness 2000). Allein auf Basis dieser Haushaltszahlen läßt sich schließen, daß der angolanische Staat - wie auch sein Gegenspieler UNITA - dem Mechanismus des Disparaten Tauschs ausgesetzt ist.

Aber das Material zum Nachweis der behaupteten Korrelation beschränkt sich nicht nur auf Haushaltsdaten. Global Witness argumentiert in seinem gut recherchierten Bericht $A$ Crude Awakening weiterhin, daß nicht nur ein Großteil des Geldes, welches die Ölgesellschaften für die vertraglich abgesicherten und von der angolanischen Regierung garantierten Bohrrechte zahlen, zur Waffenbeschaffung bestimmt ist; zusätzlich habe die Regierung durch ausländische Makler zwischen Mitte 1997 und Mitte 1998 eine ganze Reihe „ölgestützter Kredite“ erhalten und ein Großteil dieser Kredite (die insgesamt 900 Mio. US-Dollar betragen haben sollen) sei ebenfalls zum Waffenkauf verwendet worden. Die angolanische Regierung versucht sogar, den Krieg über ausländische Anleihen fortzusetzen, die über die Belastung künftiger Ölexporte abgesichert werden (Global Witness 2000). In diesen Fällen funktioniert der Disparate Tausch nicht als reiner Tauschhandel, er ist vielmehr über Geld und auch über Kredit vermittelt. So weit ich weiß, hat die angolanische Regierung sich bisher an keiner Stelle dafür entschieden, Tauschhandelsabkommen zu unterzeichnen, d.h., ohne Vermittlung durch Geld als gängigem Äquivalent Öl direkt gegen Waffen einzutauschen. Allerdings haben in einigen Fällen ausländische Firmen, von denen bekannt ist, daß sie Waffen an die angolanische Regierung geliefert haben, eine Zahlung in Form von Aktien (Anteile an Ölbohrkonzessionen) erhalten (ebd.). Da diese Aktien ihre Besitzer indirekt an dem Profit aus der Ölförderung beteili- 
gen, besteht hier eine sehr enge Korrelation zwischen Waffenimporten und Ölexporten. Ein ähnlich klares Beispiel des Disparaten Tauschs stellen die Konzessionen zur Diamantenförderung dar, die das transnationale Söldnerunternehmen Sandline International in Sierra Leone im Tausch für gelieferte Waffen erhielt (Smilie/Gberie/Hazleton 2000).

Und schließlich illustriert das Beispiel des angolanischen Öls noch zusätzlich, was die Besonderheit des disparaten Austauschs gegenüber dem Mechanismus des nur Ungleichen Tauschs ausmacht. Seit Beginn der Ölförderung 1973 war Angola über Jahre hinweg in der Lage, seine Ölproduktion auszudehnen, und eine weitere Expansion in den kommenden Jahren ist abzusehen (Global Witness 2000). Wenn der internationale Ölpreis, wie kürzlich, hoch ist, so scheinen sowohl das Land als auch andere Ölproduzenten zu gewinnen. In den Worten von Global Witness nennt sich das „doppelter Gewinn“, sowohl hinsichtlich der Wertsteigerung der Ölexporte als auch aufgrund der Tatsache, daß neue Anleihen möglich werden (ebd.). Diese Zugewinne existieren jedoch nur dem Schein nach, denn weder die Ölpreissteigerungen noch eine quantitative Produktionssteigerung tragen - unter den herrschenden Bürgerkriegsbedingungen - dazu bei, Angolas wirtschaftliche Position innerhalb des globalen kapitalistischen Systems zu verbessern. Wegen der kompletten Fesselung des Landes in die Handelsstruktur des Disparaten Tauschs sahen die Konsequenzen jeder Fördermengensteigerung oder jedes Preisanstiegs innerhalb der letzten 25 Jahre aus wie folgt: höhere Verluste eines wertvollen Rohstoffs, weitere Waffenimporte und immer weiterer wirtschaftlicher Abstieg und Zerstörung menschlicher Leben.

\section{Die Rolle von Söldnern im System des Disparaten Tauschs}

Ein wichtiges Merkmal der gegenwärtigen Bürgerkriege, die in Zusammenhang mit der Ausbeutung von Rohstoffen stehen, ist die Beschäftigung von Söldnern. Um den disparaten Austausch im Rahmen von Bürgerkriegen aufrecht zu erhalten, müssen TNCs und andere Gesellschaften, die mit dem Abbau von Rohstoffen befaßt sind, die Dienste einer verläßlichen Schutzmacht in Anspruch nehmen. Schließlich können andauernde bewaffnete Konflikte in den peripheren Ländern, in denen TNCs tätig sind, ihre Gewinne gefährden. Deshalb müssen sie sicherstellen, daß der Abbau von Rohdiamanten ohne größere Unterbrechungen fortgesetzt werden kann, und sie müssen den Schutz der Pipelines, die Rohöl von den Ölfeldern transportieren, gegen mögliche Sabotageakte durch feindliche Kräfte des laufenden Bürgerkrieges organisieren. Wo der imperialistische Tauschmechanismus von Rohstoffen gegen Waffen auf Staaten trifft, die volle Kontrolle über ihr Territorium ausüben, scheinen die Versorgungswege der TNCs (zumindest auf kurze Sicht) nicht gefährdet zu sein. Wo die TNCs jedoch, wie in Afrika, den 
Disparaten Tausch im Zusammenhang mit Bürgerkriegen aktiv ermöglicht haben, benötigen sie die Dienste professioneller Söldner zur Bewachung ihrer Versorgungswege.

Söldner, d.h. professionelle Kämpfer, die ihre militärischen Dienste gegen eine angemessene Bezahlung zur Verfügung stellen, sind natürlich kein neues Phänomen. Sie existierten bereits während des Kalten Krieges, wo sie im Sold der Kolonialmächte und deren Klientenstaaten standen und gegen erfolgreiche Guerillabewegungen eingesetzt wurden, die für nationale und soziale Befreiung kämpften. Im Zeitalter der Globalisierung haben Söldnerheere ganz eigene unternehmerische Strukturen entwickelt. Sie verfügen über eine weitaus größere Operationsfähigkeit, die sie eine Vielzahl militärischer Funktionen auf Vertragsbasis wahrnehmen läßt: von Waffentraining und Ausbildung bis hin zur Austragung von Stellvertreterkriegen. Bis in die späten 90er Jahre schossen Söldnerorganisationen wie Pilze aus dem Boden. Einige dieser Organisationen, z.B. Executive Outcomes, eine Gesellschaft mit Ursprung in Südafrika, führten gegen gute Bezahlung gleichzeitig Operationen für die Regierungen mehrerer afrikanischer Länder aus. In Anbetracht ihres kommerziellen Charakters fällt es Söldnerorganisationen nicht schwer, je nach Interessenlage ihrer Auftraggeber oder der angebotenen Entlohnung, die Loyalitäten zu wechseln.

Während in der Vergangenheit Söldner häufig als Kreuzfahrer gegen den Kommunismus antraten, ist dies im Zeitalter der Globalisierung und des Systems des Disparaten Tauschs nicht mehr möglich, die gegenwärtigen Söldnertätigkeiten besitzen nicht mehr den geringsten ideologischen Anstrich. Die Firma Gurkha Security Guards beispielsweise soll Schutzfunktionen für das britische Bergwerksunternehmen Lonrho in Mosambique ausgeübt haben, Sandline International, ein weiteres aus Großbritannien stammendes Söldnerunternehmen, unterhält angeblich enge Verbindungen mit Tochterfirmen der Diamantenindustrie (Smilie/Gberie/Hazleton 2000). Ein besonders deutliches Beispiel zur Illustration der unideologischen Natur heutigen Söldnertätigkeit sind die Aktivitäten von Executive Outcomes in Angola. Sie hatte zunächst mit der UNITA zusammengearbeitet, 1992 hatte sie dann im Auftrag der angolanischen Regierung Ölfelder der TNCs geschützt und Mitte der 90er Jahre eroberte sie von der UNITA einige Diamantenminen zurück, welche De Beers mittlerweile lieber unter der legalen Herrschaft des angolanischen Staats bewirtschaften wollte (Jung 2000: 14).

Eine Veröffentlichung des Internationalen Komitees des Roten Kreuzes in den späten 90er Jahren unter dem Titel Krieg, Geld und Überleben faßt die enge Verbindung zusammen, die heute zwischen Rohstoffen, Bürgerkriegen in Afrika und den Operationen von Söldnerheeren besteht. Die IKRKVeröffentlichung besagt, daß in den 90er Jahren in Afrika angesichts der Schwäche der meisten staatlichen Armeen die „Nachfragebedingungen“ für 
die Privatisierung von Kriegen besonders gut waren. Während die regulären Armeen schwach waren, verfügten die Regierungen häufig über Zugang zu natürlichen Ressourcen, „die zur Bezahlung von Außenstehenden genutzt werden konnten, damit diese ihr militärisches Wissen zur Verfügung stellen“. Daher „gibt es auf jeden Fall eine sehr starke Korrelation“ zwischen natürlichen Ressourcen und dem Einsatz von Söldnerheeren (Nossal 200: 37). Im Umkehrschluß wird weiter gefolgert, daß „private militärische Organisationen in afrikanischen Konflikten nicht zum Einsatz kommen, wenn keine Ressourcen auf dem Spiel stehen“ (ebd.). Damit bestätigt die IKRK Veröffentlichung, daß Söldnerheere zum militärischen Flügel der imperialistischen Handelsstrukturen des Ungleichen und Disparaten Tauschs geworden sind.

\section{Disparater Tausch und die dauerhafte Abschöpfung des afrikanischen Kontinents}

Das Beispiel Angola illustriert besonders plastisch die Bedeutung des Disparaten Tauschs. Als theoretisches Konzept wurzelt der Disparate Tausch in der älteren Theorie des Ungleichen Tauschs, welche sich mit der ständigen Abschöpfung der Ressourcen südlicher, peripherer Volkswirtschaften beschäftigt: aufgrund ihrer Abhängigkeit vom Rohstoffexport und der beständigen Verschlechterung ihrer terms of trade. Disparater Austausch wie er sich im angolanischen Beispiel darstellt, führt aber nicht nur zu einer Abschöpfung von Ressourcen, zum Transfer des Reichtums von Süd nach Nord, sondern vor allem zu Verarmung und Zerstörung aufgrund des Gebrauchs von Waffen, die von den Kriegsparteien mit Einkünften bezahlt werden, die sie aus dem Verkauf von Diamanten, Öl und anderem einheimischen Reichtum erzielten. Die Zahlen, die der Human Development Report des UNDP angibt, unterstreichen, daß, obwohl das Land reich mit natürlichen Ressourcen ausgestattet ist, das angolanische Volk heute unter massiver Armut und Verelendung leidet (Global Witness 1998). ${ }^{5}$

Aber das angolanische Beispiel steht bei weitem nicht allein. Disparater Tausch kennzeichnet genauso die Vorgehensweise konkurrierender Parteien im westafrikanischen Sierra Leone. Die Weltpresse hat der Tatsache viel Aufmerksamkeit geschenkt, daß die Rebellenbewegung des Landes, die R.U.F., sich der grauenvollsten Methoden bedient hat, um im Bürgerkrieg die Oberhand zu gewinnen. Und es ist gleichermaßen kein Geheimnis, daß die R.U.F. ihren Krieg mittels Diamanten finanziert, die über Liberia und andere Nach-

5 Global Witness bezieht sich auf Daten aus dem Human Development Report der UNDP von 1997: Angola nahm unter 175 aufgelisteten Ländern den 157. Platz ein; mehr als 82\% der Menschen leben in „absoluter“ oder „relativer“ Armut, nur 35\% der Bevölkerung hatten Zugang zur Gesundheitsversorgung, und die Kindersterblichkeitsrate für Kinder unter 5 Jahren lag bei 320 von 1000 ! 
barländer geschmuggelt werden. Aber auch die Regierungsseite in Sierra Leone verläßt sich auf Rohstoffe zur Kriegsfinanzierung, nur daß in diesem Falle die Verkäufe nicht allein auf Diamanten beschränkt sind. Wie erst kürzlich in Le Monde Diplomatique enthüllt wurde, treiben rivalisierende Unternehmen, die sich um Abbaukonzessionen drängeln, die militärischen Kampagnen beider Seiten voran (Perez 2000: 4/5). Und es besteht keinerlei Mangel an Beweisen, daß auch in diesem Fall der Tausch von Rohstoffen gegen Waffen das Wohl der Bevölkerung des Landes unterminiert hat. Sierra Leone ist ein weiteres Land, das herangezogen werden kann, um die Folgen des Disparaten Tauschs zu illustrieren.

Die These vom Ungleichen Tausch, die seit 1949 zunächst aufgrund empirischer Untersuchungen unter Federführung der Vereinten Nationen, und später durch die theoretischen Bemühungen marxistisch orientierter Ökonomen (Emmanuel 1972; Amin 1974) erhärtet wurde, beinhaltete, daß „periphere“ Länder einem beständigen Prozess des Ressourcenabflusses unterworfen seien. Angesichts der Tatsache, daß Afrikas Rolle innerhalb des Welthandels vor allem vom Export von Diamanten, Öl, Kobalt, Bauxit und anderen Rohmaterialien abhängt, ist der Kontinent viel stärker von den wechselnden Terms of Trade von Rohstoffen betroffen als andere Kontinente und Regionen des Südens. Es ist kein Zufall, daß die Organisation Afrikanischer Einheit (OAU) 1990, als die USA und andere imperialistische Staaten starke Anstrengungen unternahmen, um die Debatte über Ungleichen Tausch zu beenden, eine Resolution verabschiedete, die an die Welt appellierte, die schwerwiegenden Terms of Trade Shocks zur Kenntnis zu nehmen, unter denen die afrikanischen Volkswirtschaften litten (The Netherlands Ministry of Foreign Affairs: $19 / 20)^{6}$

Trotzdem haben die afrikanischen Länder weitaus größere Rückschläge durch Disparaten als durch Ungleichen Tausch erlitten. Wie eine Krake hat sich das System des disparaten Austauschs in den letzten Jahren weiter ausgebreitet, und hält inzwischen auch den Kongo mit seinen großen natürlichen Ressourcen in seinen Tentakeln gefangen. ${ }^{7}$ Die in Angola, Sierra Leone, dem Kongo und anderen reich ausgestatteten afrikanischen Ländern gemachten Erfahrungen beweisen, daß das System des Disparaten Tauschs nicht nur für den Transfer des Reichtums vom Süden in den Norden steht. Denn Hand in Hand mit dieser wirtschaftlichen Ungerechtigkeit werden den Menschen in Afrika Verletzungen zugefügt, die nichts anderes als ein kollektives kriminelles Unrecht darstellen. Denn während wertvolle Rohstoffe zu produktiven

6 Henk Overbeek (2000) zufolge fiel das Preisniveau für Primärprodukte, die 80\% des afrikanischen Exports ausmachen, zwischen 1997 und 1999 um ein Viertel.

7 Internationale Kampagnen zum Thema Rohstoffe und Kriege in Afrika haben sich bisher wenig mit den Kriegsursachen im Kongo befaßt, obgleich das Land zweifelsohne ein strategisches Ziel transnationaler Bergwerksgesellschaften ist (vgl. Baracyetse o.J.). 
und zu Konsumzwecken in den Norden transportiert werden, werden die Mittel der Zerstörung, die im Austausch für diese Rohstoffe nach Afrika importiert werden, in einer ständig wachsenden Reihe von Bürgerkriegen und regionalen Auseinandersetzungen „konsumiert“. Die Folgen des Disparaten Tauschs bestehen also nicht nur in Verlusten, sondern in einer unermeßlichen Zerstörung. Obwohl einige nahöstliche Länder trotz des Disparaten Tauschs eine gewisse Entwicklung erfahren haben, ist die Bilanz für die afrikanischen Länder durchweg negativ.

\section{Schlußfolgerung: Afrika im System Disparaten Tauschs}

Um Afrikas Stellung innerhalb des Systems des Disparaten Tauschs besser zu verstehen, empfiehlt sich ein kurzer Vergleich zwischen der Anwendung dieses Handelssystems im Nahen Osten einerseits und im afrikanischen Kontext andererseits. Die Unterschiede zwischen den beiden Regionen sind immens. Obgleich man in beiden Fällen einen Austausch von Rohstoffen gegen Waffen feststellen kann, variiert doch der konkrete Kontext, innerhalb dessen sich dieser Disparate Tausch abspielt, signifikant. Im Nahen Osten behalten die Staaten die Kontrolle über die entscheidende natürliche Ressource der Region, das Öl, und das Handelssystem des Disparaten Tauschs hat hauptsächlich zwischenstaatliche Konflikte hervorgebracht. Im Gegensatz dazu ist das Muster des Disparaten Tauschs in Afrika in erster Linie über Bürgerkriege institutionalisiert worden, die sich zwar in keiner Weise unblutiger präsentieren als die im Nahen Osten ausgefochtenen Golfkriege, aber nichtsdestotrotz einer völlig anderen Dynamik unterliegen.

Ein weiterer, damit zusammenhängender Unterschied beinhaltet das Ausmaß der Waffenlieferungen in die entsprechenden Regionen. Während der Nahe Osten schon lange einen großen Anteil am Fluß von Waffen und Rüstungssystemen aus dem Norden in den Süden hatte, ist der afrikanische Anteil am internationalen Waffenhandel vergleichsweise klein. Einige Länder gelten zwar als besonders kaufkräftige Kunden, besonders Südafrika, das 1999 Waffenimporte im Wert von 5 Mrd. US-Dollar orderte (Hagelin/Wezeman and Wezeman 2000) ${ }^{8}$, und Angola, dessen Einkaufspraxis bereits erläutert wurde. Allerdings verfügen die meisten Staaten und Konfliktparteien über so geringe Finanzmittel, daß sie sich lediglich den Erwerb kleiner Waffen, Munition und

8 „1999 unterzeichnete Südafrika Verträge im Wert von 29,99 Mrd. Rand (etwa 5 Mrd. USDollar) für Waffenlieferungen ausländischer Firmen. Im Gegenzug haben sich diese Firmen verpflichtet, über Gegenkäufe und Investitionen in Südafrika Geschäfte im Wert von insgesamt 104 Mrd. Rand (etwa 17,3 Mrd. US-Dollar) zu machen, aber solche Kompensationspläne können sich über eine Periode von 10 bis 15 Jahren erstrecken und werden möglicherweise nie umgesetzt. Trotz der hohen Kompensationen, die man Südafrika bot, entschied es sich allerdings, die Entscheidung über zwei Drittel der geplanten Käufe für die Luftwaffe auf einen späteren Zeitpunkt zu verschieben“ (ebd.). 
Minen leisten können. Während das Sipri Jahrbuch 2000 darüber spekuliert, daß das Gewicht des nahöstlichen Marktes in Zukunft noch anwachsen könne, ist Afrikas Anteil seit den 70er Jahren in quantitativer Hinsicht bis hin zur Unwesentlichkeit geschrumpft. Manche Quellen datieren den Anteil des Kontinents am weltweiten Waffenimport auf 2,3\% und weniger (US Department of State 2000).

Das hat unter anderem zur Folge, daß sich die Profile der transnationalen Konzerne, die von der Praxis des Disparaten Tauschs profitieren, in den beiden Regionen erheblich unterscheiden. Im Fall des Nahen Ostens gehören sowohl die größten Hersteller von Vernichtungswaffen aus dem Norden, d.h. Rüstungsproduzenten mit Sitz in den USA, Großbritannien, Frankreich etc., als auch die „sieben Schwestern“, d.h. die mächtigsten Ölgesellschaften, dazu. Im Falle Afrikas beschränken sich die Nutznießer des Disparaten Tauschs hauptsächlich auf Rohstoffe ausbeutende Gesellschaften wie die Monopolgesellschaft auf dem Diamantensektor, De Beers, und die Ölgesellschaft Elf Aquitaine. Die direkten Nutznießer des Waffenhandels umfassen dagegen viele Zwischenhändler, Luftfahrtgesellschaften und mittelständische Unternehmen (viele von ihnen mit Sitz in Osteuropa) (Wood/Peleman 1999), und die weniger großen Rüstungsunternehmen. In Anbetracht der ungeheuren Verarmung des Kontinents gibt es in Afrika nur wenige Kunden für militärische TNCs .

Trotzdem wäre es falsch anzunehmen, daß Afrika nur eine unbedeutende Rolle innerhalb der Struktur des Disparaten Tauschs einnehmen würde. Ganz im Gegenteil! Die Erfahrungen, die hier mit dem Disparaten Tausch gemacht wurden, hauptsächlich im Zusammenhang mit den vielfältigen Bürgerkriegen des Kontinents, zeigt den habgierigen transnationalen Akteuren, die den Weltmarkt dominieren, ein weitgefächertes Betätigungsfeld. Denn wo sich der methodische - legale wie illegale - Parallelexport ein und desselben Rohstoffs über verfeindete Kräfte in Bürgerkriegen als zunehmend effizient herausstellt, wo die Mechanismen zum Schutz von Abbaugebieten und Pipelines durch Söldner zunehmend ausgefeilter werden, wo sich diesem System immer weitere Rohstoffe hinzugesellen, und wo die Ideologie des Freihandels ihre Vorherrschaft zunehmend behauptet - da drängt sich den TNCs die Nachbildung eben dieses Handelssystems auch in anderen geographischen Regionen der Welt von selbst auf. Ganz gleich wie negativ die Bilanz afrikanischer Bürgerkriege für die Bewohner des eigenen Kontinents ausfallen mag - vor dem Hintergrund seiner Bürgerkriege hat sich der Disparate Tausch als modernes System der Ausbeutung für all jene bewährt, die die kapitalistische Weltwirtschaft beherrschen. 


\section{Literatur}

Amin, Samir (1974): Accumulation on a World Scale. A Critique of the Theory of Underdevelopment, in: Monthly Review Press, New York/London.

Baracyetse, Pierre (o.J.): L'Enjeu Geopolitique des Transnationales Minieres au Congo, (publication of SOS Rwanda-Burundi).

Carbonnier, Gilles; Fleming, Sarah (2000): War Money and Survival, Genf, Schweiz.

Collier, Paul (2000): Economic Causes of Civil Conflict and Their Implications for Policy, in: World Bank Development Research Group, Washington D.C.

Emmanuel, Arghiri (1972): Unequal Exchange. A Study of the Imperialism of Trade, in: Monthly Review Press, New York/London.

Global Witness (1998): A Rough Trade. The Role of Companies and Governments in the Angolan Conflict, London, U.K.

- (2000): A Crude Awakening. The Role of the Oil and Banking Industries in Angola's Civil War and the Plunder of State Assets, London, U.K.

Hagelin, Bjorn; Wezeman, Pieter D. (2000); Wezeman, Siemon T.: Transfers of Major Conventional Weapons, in: 2000 Yearbook of the Stockholm International Peace Research Institute (Sipri).

Human Rights Watch, Angola (1994): Arms Trade and Violations of the Laws of War since the 1992 Elections, in: Human Rights Watch Arms Project/Human Rights Watch/Africa Nov. 1994.

Jung, Anne (2000): Angola im Dritten Jahrzehnt des Krieges. Verkauf von Diamanten und Öl an den Norden finanziert den Krieg, in: Analyse \& Kritik, No. 437, April 13, 2000.

Mandel, Ernest (1974): Marxist Economic Theory, London, U.K.

Nossal, Kim Richard (2000): Bulls to Bears: The Privatisation of War in the 1990's, in:

Overbeek, Henk (2000): Afrikas Heeft Niets te Verwachten van Globalisering', Handelsblad, 15. August.

Perez, Andres (2000): Guerre et Diamants en Sierra Leone, in: Le Monde Diplomatique, June 2000, p. $4 / 5$.

Roche, Marc (2000): La De Beers a Mis Fin a Dernier Vrai Cartel au Monde, in: Le Monde, July 16/17, 2000, 16.

Shiva, Vandana (1998): Biopiracy, London, U.K.

Smilie, Ian; Gberie, Lansana; Hazleton, Ralph (2000): The Heart of the Matter. Sierra leopne, Diamonds and Human Security, Ottawa, Canada.

The International Institute for Strategic Studies (IISS) (2000): Diamnonds and Conflict. Strategies for Control, Strategic Comments, in: IISS, Vol.6, Issue 4, May 2000.

The Netherlands Ministry of Foreign Affairs, Global Coalition for Afrika (k.A.): Documents on: Development, Democracy and Debt, Den Haag, Niederlande.

US Department of State, Bureau of Arms Control (2000): World Military Expenditures and Arms' Transfers, Washington DC, USA.

Wood, Brian; Peleman, Johan (1999): The Arms Fixers - Controlling the Brokers and Shipping Agents (International Peace Research Institute PRIO), Oslo. 


\section{Anne Jung}

\section{Das Geheimnis einer faszinierenden Leidenschaft - Kriegsdiamanten Die Internationale Kampagne Fatal Transactions}

Über die skrupellose Ausbeutung von Bodenschätzen in Afrika informiert die internationale Kampagne Fatal Transactions. Von medico international ${ }^{1}$ gemeinsam mit den europäischen Partnerorganisationen global witness ${ }^{2}$, NOVIB (Netherlands Organization for International Development Cooperation) und NIZA (Netherland Institute on Southern Africa) gegründet, hat es sich die Kampagne zur Aufgabe gemacht

- über Geschäfte, die Kriege in Afrika in Gang halten, aufzuklären;

- internationale Unternehmen zum Rückzug aus dem schmutzigen Rohstoffhandel zu bewegen;

- Konzerne, die in den vergangenen Jahrzehnten an dem illegalen Handel profitiert haben, für die Beseitigung der Kriegsschäden und die Entschädigung der Opfer verantwortlich zu machen.

Von den milliardenschweren Geschäften mit Bodenschätzen wie Diamanten, Öl und neuerdings auch Coltan profitieren internationale Unternehmen genauso wie korrupte Politiker, Warlords, Waffenhändler und private Söldnerfirmen. Sie konkurrieren und kooperieren miteinander (je nach Bedarf) und sorgen so für die Weiterführung der Kriege.

Ein zentrales Thema von Fatal Transactions ist der Diamantenhandel in Angola, in Sierra Leone und in der DR Kongo.

Beispiel Angola. Seine Bodenschätze machen Angola zum potentiell viertreichsten Land der Welt. Mit dem Schmuggel der reichen Diamantenvorkommen finanziert die angolanische Rebellenbewegung UNITA (Nationalunion für die volle Unabhängigkeit Angolas) seit fast 30 Jahren ihren

1 Die Frankfurter Hilfsorganisation medico international initiierte die „Internationale Kampagne zum Verbot von Landminen“ und erhielt 1997 für dieses Engagement mit gemeinsam Partnerorganisationen den Friedensnobelpreis.

2 Global witness machte sich in den vergangenen Jahren mit aufsehenerregenden Studien zum Diamanten- und Ölhandel in Angola einen Namen und hat maßgeblich zum Zustandekommen des sog. „Kimberley-Prozesses“ beigetragen, in dem Vertreter aus 38 Regierungen, der Hohe Diamantenrat, UN-Vertreter und Nichtregierungsorganisationen einen ausführlichen Richtlinienkatalog erarbeiten, der die Einhaltung des Embargos sicherstellen soll. 
Krieg gegen die MPLA-Regierung (Volksbewegung für die Befreiung Angolas). Die Regierung ihrerseits finanziert den Krieg maßgeblich durch den Ölhandel. Die Kontrolle der reichen Bodenschätze wurde immer mehr zur Ursache des Krieges. Die Waffenlieferungen aus Bulgarien, den USA und Russland garantieren logistisch die Weiterführung des Krieges auf beiden Seiten. Der Krieg hat über 500000 Angolanerinnen und Angolanern das Leben gekostet, zehntausende sind nach Unfällen mit Landminen verkrüppelt. Beide Konfliktparteien terrorisieren die Zivilbevölkerung und plündern sie aus. Die mutigen Friedensbemühungen der angolanischen Zivilgesellschaft werden immer wieder zunichte gemacht. Soweit die dramatischen Zahlen, die sich weiter fortführen und auf andere Länder in Afrika ausdehnen ließen.

Jahrzehntelang hat der Marktführer De Beers bedenkenlos die Steine aus Kriegsregionen gekauft, so auch die der UNITA in Angola, die mit ihrem jährlichen Verdienst von ca. 500 Millionen US\$ ihre Waffenkäufe finanziert. Noch heute handeln viele Diamantenkonzerne mit Steinen aus Krisengebieten, auch unter den Vorräten von De Beers finden sich viele Kriegsdiamanten.

Die Diamantenindustrie lebt in doppelter Hinsicht vom symbolischen Wert der Diamanten. Zum einen verkörpern die Steine die Stereotypen der unvergänglichen Liebe, des Glücks und des Wohlstands - jeder kennt die großen Anzeigen auf Plakatwänden und in den Hochglanz-Zeitschriften. Zum anderen lebt die Branche von dem selbstgeschaffenen Mythos, die Steine seien selten. De Beers hält Vorräte im Wert von ca. 2,5 Mrd. US\$ zurück, um den Preis künstlich hoch zu halten. Die Kunden bezahlen Millionen für Diamanten, deren Wert jedoch ausschließlich in der Vorstellung ihrer Käufer und Käuferinnen existiert: "Nimm den Steinen ihre symbolische Kraft und der gesamte Markt bricht zusammen." (Stan Correy, Diamantenexperte). Aus diesen Gründen hat die Diamanten-Industrie Angst vor einem Imageschaden, die Fatal Transactions nutzen konnte.

Als Fatal Transactions im Herbst 1998 begann, öffentlich über die Schattenseiten der begehrten Steine zu berichten, reagierte die Diamantenbranche nervös. Um drohenden Umsatzverlusten zuvorzukommen, versprach De Beers in einem Brief an medico, ab sofort auf den Handel mit Kriegsdiamanten verzichten zu wollen. Auch der World Congress of Diamonds beschloß im Sommer 2000, in Zukunft keine Schlupflöcher für Kriegsdiamanten zu lassen. Unterzeichnet wurde die Resolution auf oberster Ebene: vom Internationalen Diamantenherstellerverband und dem Weltbund der Diamantenbörsen.

Bald wurde jedoch klar, dass das bestehende UN-Embargo gegen Kriegsdiamanten Schlupflöcher läßt: Die angolanische UNITA z.B. verkauft über ein Netz von Händlern und Gesellschaften weiterhin jährlich Diamanten im Wert von mind. 4 Millionen US\$ auf den Märkten in Europa. 
Fatal Transactions fordert daher:

- Alle Diamantenkonzerne, Händler und Regierungen sollen ihren Handel transparent gestalten.

- Die am Diamantenhandel beteiligten Akteure müssen verpflichtet werden, mit Prüfinstanzen - unabhängigen Experten - zusammenzuarbeiten.

- Diamantenhändler, die sich nicht an das Embargo halten, sollen auf eine schwarze Liste gesetzt und von allen Diamantenbörsen ausgeschlossen werden.

- Die Kommission darf die Vergabe eines Herkunftszertifikates nicht ausschließlich vom Exportland abhängig machen. Bislang gilt das Ankaufland gleichzeitig als Herkunftsland.

In einem Brief an den Bundesverband der Juweliere fragte medico nach, wie sie sicherstellen können, dass keine Diamanten nach Deutschland gelangen, deren Herkunft nicht einwandfrei geklärt ist. Eine Antwort steht bis heute aus. Mit einer Postkartenaktion sollen Juweliere im gesamten Bundesgebiet gedrängt werden, sich im Rahmen ihrer Möglichkeiten um die Durchsetzung des Embargos zu bemühen.

Fatal Transactions geht weit über die Forderung nach Einhaltung des Embargos hinaus. Die Kampagne sieht es als ihre Aufgabe an, die ökonomischen Interessen, die Regierungen oder die Industrie zum Einlenken bewegen, in die Diskussion zu bringen. Zwei Beispiele:

- Die neue Strategie von De Beers ist auch von dem strategischen Interesse geleitet, den Preis der Diamanten zu stabilisieren. Und der ist gefährdet, seit der informelle Sektor - der illegale Markt schwer kontrollierbarer Diamantenschmuggler und Kleinförderer -auf den Markt drängt. De Beers befürwortet die Einhaltung des Embargos und versucht damit, konkurriende Förderer mit Hilfe von Standards vom Markt zu drängen, die andere Händler nicht erfüllen können.

- Die Bemühungen der US-amerikanischen und kanadischen Regierung, die Durchsetzung des Embargos zu erreichen, erscheinen in einem veränderten Licht, wenn man die dahinter stehenden ökonomischen Interessen betrachtet: US-amerikanische Konzerne haben 8 Milliarden DM in Angola investiert, das derzeit $7 \%$ des amerikanischen Öl-Bedarfs deckt. Auf 16\% soll dies bis zum Jahr 2005 gesteigert werden. So lag es nahe, auch politisch die Seiten zu wechseln und nach über 20 Jahren die Unterstützung der UNITA aufzugeben. ${ }^{3}$

3 Die USA finanzierten den Krieg der UNITA mit 60 Millionen US\$ jährlich (New York Times, 14.01.01) 
Um sich nicht von der Industrie funktionalisieren zu lassen, betont Fatal Transactions die unmittelbare Wirkung und die generelle Bedeutung des Handels mit den kriegszerrütteten Ländern in Afrika für die Fortführung der Kriege, damit sich die Konzerne aus diesen Ländern zurückziehen und gezwungen werden, Entschädigung für die Beseitigung der Kriegsschäden und die Opfer zu zahlen. Ein wichtiges Ziel ist dabei die Schaffung von juristischen Präzedenzfällen.

Wären die internationalen Unternehmen bereit, die Wirtschaftsbeziehungen zu den Kriegsherren aufzugeben, würde das Geschäft mit der Gewalt unrentabel. Dafür wird sich Fatal Transactions mit einer kritischen Öffentlichkeitsarbeit weiter einsetzen.

\section{Literatur}

global witness: A rough trade. London 1998

Institute for Security Studies (ISS) (Hg.): Angolas war economy. The role of oil and diamonds. Pretoria 2000

Anne Jung: Harte Männer schenken harte Steine. In: Wissenschaft und Frieden 4/2000.

Siegfried Pater: Blutige Diamanten. Lamuv-Verlag. Köln 2001

\section{Internationale Tagung zur Bürgerkriegsökonomie vom 18.-21.10.01}

veranstaltet von medico international und der Ev. Akademie Hofgeismar

Die Tagung untersucht die Reproduktionsmechanismen und Funktionsweisen anhand von Länderbeispielen (Sierra Leone, Angola, Kolumbien, Kambodscha) und entlang von Themenfeldern wie der Rolle von Söldnern, Waffenhändlern und Hilfsorganisationen.

ReferentInnen: Amy Smythe (Sierra Leone), Peter Lock (Hamburg), Klaus Schlichte (Hamburg), Philippe Le Billion (GB), William Reno (USA), Khareen Pech (Südafrika), Dario Azzelini (Berlin), Wolf-Christian Paes (Bonn)

Weitere Informationen zu der Kampagne und der Tagung bei medico international. Obermainanlage 7, 60314 Frankfurt, Tel. 0049 (0)69 9443827 www.medico.de 